\title{
Gas Pressure Induced Porosity of CYTOP Polymer. A Positron Study
}

\author{
B. Zgardzińska*, T. Goworek, M. ŚniegockA, R. Zaleski \\ Institute of Physics, Maria Curie-Skłodowska University, pl. M. Curie-Skłodowskiej 1, 20-031 Lublin, Poland \\ AND G. DLUBEK
}

ITA Institut für Innovative Technologien, Kóthen/Halle, D-06120 Lieskau, Germany

\begin{abstract}
The pore formation in CYTOP polymer under high pressure of argon or nitrogen gases was investigated by positron annihilation spectroscopy. In the range 0-70 $\mathrm{MPa}$, formation of large open pores was observed. After reduction of pressure to normal one, the free volumes inside the polymer structure remained enlarged. The structure of CYTOP can be restored by applying the temperature over $470 \mathrm{~K}$ at normal pressure.
\end{abstract}

DOI: 10.12693/APhysPolA.125.782

PACS: 78.70.Bj, 74.62.Fj

\section{Introduction}

There are many publications devoted to modifying the void sizes (free volumes) in the structure of polymers; in particular to expanding them by exposure to a high gas pressure [1-7]. The size modification was usually investigated by positron annihilation lifetime spectroscopy (PALS). The pressures applied to modify the polymer structure were limited to $2 \div 5 \mathrm{MPa}$, particular attention was paid to $\mathrm{CO}_{2}$ atmosphere [5-7]. In all papers quoted above the samples were exposed to a high gas pressure, but the PALS measurements were performed after its reduction to normal one; the pressures required to obtain the swelling effect were rather low, not exceeding $5 \mathrm{MPa}$, except Ref. [6] (to $24 \mathrm{MPa}$ ). This paper presents the results of measurements performed on the samples being still under pressure when their PALS spectra were collected. Moreover, much broader range of pressure, over $100 \mathrm{MPa}$, was applied. As an object of study we have chosen CYTOP, a heterocyclic-ring-containing polymer (from Asahi Glass Co.) with glass transition point $T_{\mathrm{g}}=377 \mathrm{~K}$. This polymer is amorphous, exceptionally transparent in a broad range of wavelengths from $200 \mathrm{~nm}$ up to near IR, applied for short range lightguides.

The PALS spectra of CYTOP taken as a function of temperature or pressure with no gas exposure [8] show only a monotonous decrease of the lifetime and intensity with an increase of pressure. Like in many other polymers, swelling of free volumes after the exposure to $\mathrm{CO}_{2}$ at the pressure up to $5 \mathrm{MPa}$ was observed in the whole range of temperatures below the glass transition point [7].

\section{Experimental}

The PALS spectra were measured using a standard fast-slow delayed coincidence spectrometer. The sam-

\footnotetext{
${ }^{*}$ corresponding author
}

ples of polymer had the form of discs cut from the $1 \mathrm{~mm}$ thick sheet; the positron source ${ }^{22} \mathrm{Na}$ in a Kapton foil envelope, $8 \mathrm{~mm}$ in diameter, was inserted between them. The sample-source sandwich was pressed together and placed in the pressure chamber of the gas compressor (Unipress U-11). Before the measurements, the chamber was flushed with argon to remove the traces of air (oxygen). The temperature of the sample was controlled with an accuracy of $\pm 0.1 \mathrm{~K}$ by the Peltier cell and a heating coil contacted with an aluminum block clamping the measurement chamber. The spectra were analyzed as a sum of exponential components (if not stated otherwise) convoluted with the instrumental resolution curve, using the LT v.9 program [9]. The resolution curve was approximated by a single Gaussian, 220 ps FWHM. Some spectra, collected with high statistics, were processed using the MELT procedure [10], which finds a quasi-continuous distribution of lifetimes without an a priori assumption of the number of components. Absorption of gamma radiation in the walls of the pressure chamber and in the thick aluminum block reduced the rate of data collection, so an accumulation of one spectrum containing $10^{6}$ events required $3 \mathrm{~h}$. The statistics in the spectra for MELT analysis was by order of magnitude higher.

\section{Results and discussion}

In Refs. $[7,8]$, the CYTOP spectra without gas intrusion were analyzed assuming the presence of one component belonging to $o$-Ps but with the lifetime distribution of log-Gaussian shape. However, the MELT processing shows that the $o$-Ps lifetimes in the samples without previous treatment by high pressure represent not a single broad component, but two distinctly different, quasi-discrete ones, $\approx 1.5 \mathrm{~ns}$ and $\approx 4 \mathrm{~ns}$. We will call the free volumes producing these two components "natural" ones. This form of the spectrum, containing two $o$-Ps components, was assumed in the further processing of the data. It seems interesting that the lifetime of $4 \mathrm{~ns}$ 
corresponds, according to the Tao-Eldrup model [11], to spherical voids of the radius $0.44 \mathrm{~nm}$ (or channels of the radius $0.38 \mathrm{~nm}$ ), thus, the free volumes in "no pressure" CYTOP samples are larger than e.g. the typical bubble which can be worked up in liquids by positronium against the surface tension.

The PALS spectra of CYTOP as a function of argon pressure were measured at $298 \mathrm{~K}$. Expecting the appearance of porosity, we processed the PALS spectra using the LT program assuming the existence of five discrete components; the three longest ones belonging to ortho-Ps, the others - to the annihilation of free positrons and the decay of para-Ps. The only constraint imposed initially on the analysis was fixing the para-Ps lifetime as 127 ps (an average of lifetimes found in a set of spectra).

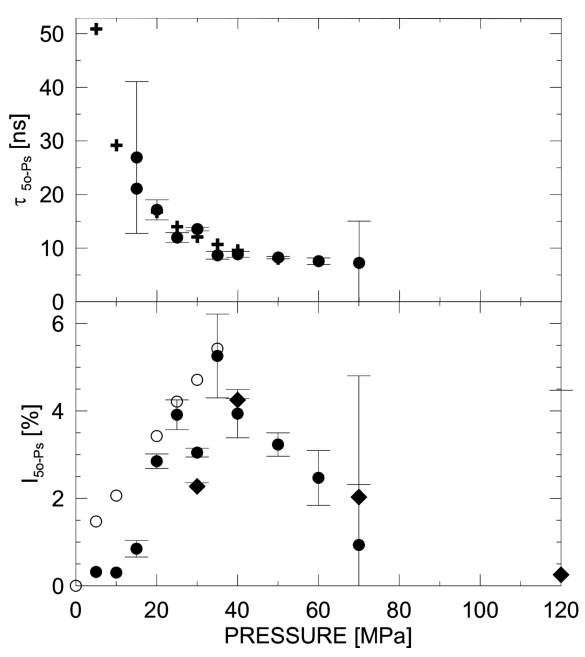

Fig. 1. The pressure dependence of $\tau_{5}$ lifetime and $I_{5}$ intensity in CYTOP in argon atmosphere. Full symbols - pressure up, open symbols - pressure down. Crosses denote the lifetime of $o$-Ps in gaseous argon, diamonds - $I_{5}$ intensity in nitrogen atmosphere.

The results are shown in Figs. 1 and 2. The new longest-lived component is visible already at the pressure $5 \mathrm{MPa}$. In all cases, when the fifth component was found, its lifetime was very close to that of gaseous argon (see the top of Fig. 1), and, in the final spectrum processing, the values of that lifetime were chosen to be equal to those obtained in a separate measurement when the measuring chamber was filled with gas only. The similarity of the $\tau_{5}$ lifetime to that measured in pure argon indicates a weak Ps interaction with the walls of a void and thus a large size of created free volumes. Due to the great number of fitting parameters and moderate statistics, the uncertainties of the lifetime and intensity were rather large. The highest intensity of the new long component was about $(4 \div 5) \%$ at $35 \mathrm{MPa}$, and then diminished with pressure; at $70 \mathrm{MPa}$ it amounts to $1 \%$ only. Extrapolating the $I_{5}$ data one can presume that in higher pressures this intensity is close to zero. An additional difficulty appeared while processing the spectra in the range $(80 \div 120) \mathrm{MPa}$ : the $o$-Ps lifetime in argon at

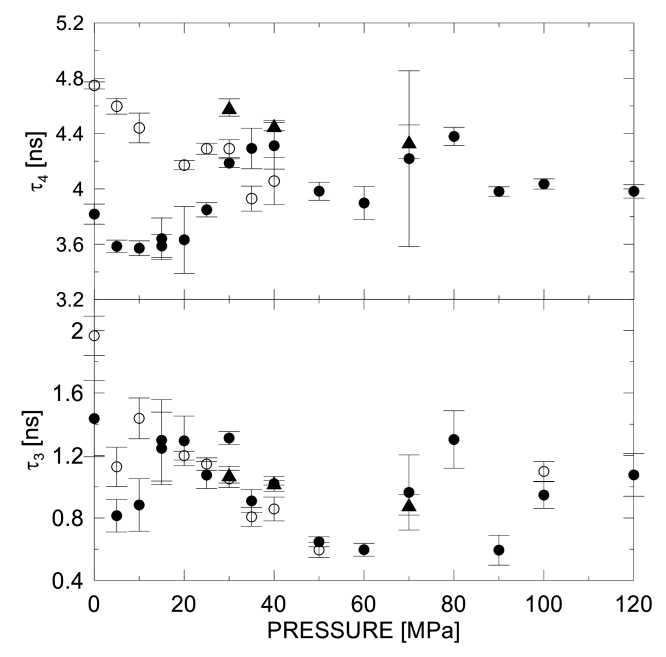

Fig. 2. The pressure dependence of the lifetimes $\tau_{3}, \tau_{4}$ in CYTOP in argon atmosphere obtained by the LT processing. Full circles - pressure up, open circles pressure down. Triangles are for CYTOP in the atmosphere of nitrogen.

these pressures is $(4.0 \div 4.5)$ ns, i.e. coincident with the $\tau_{5}$. Thus, the experimental points in Fig. 2 for $p \geq 80 \mathrm{MPa}$ were obtained for the 4-component analysis.

The fraction of positronium belonging to the new component can be produced in the solid and transferred to big free volumes, or directly in the gaseous medium. One can suppose that argon penetrates easily into the polymer, producing channels (the radius $0.4 \mathrm{~nm}$ is at least twice of the molecular sizes of argon or nitrogen). The results of our measurements, however, do not give any information about the sizes and the concentration of pore-like structures, because in this case the lifetime $\tau_{5}$ characterizes rather the gas medium, not the sample. After the reduction of pressure to normal one, this component is not detectable, thus, the free volumes produced in the sample by gas pressure are open. The tendency of the intensity $I_{5}$ to approach zero at the reduction of the pressure means also that all Ps forming this component are produced in the gas, transitions from the solid to the pores is negligible, perhaps with the exception of the lowest pressures. At 5 and $10 \mathrm{MPa}$, the intensities $I_{5}$ were found larger than these observed at rising pressure. It can be an indication that the Ps formation probability depends slightly on the structure of the surrounding medium, which can be modified by the pressure treatment. In the range from $25 \mathrm{MPa}$ down to zero, the lifetime $\tau_{4}$, being the main characteristics of the "natural" free volumes in the polymer structure, is longer than in the run with rising pressure; the pressure introduces a permanent damage of the polymer structure. The same effect was reported by Dlubek et al. [7]. It is interesting that the lifetime of the main "natural" $o$-Ps component, $\tau_{4}$, is almost insensitive to the applied pressure (at least in the range below $120 \mathrm{MPa}$; at $320 \mathrm{MPa}$ it diminishes to $3.4 \mathrm{~ns}$ ). Figure 3 shows the MELT spectrum of the 
longest-lived components in CYTOP sample at normal pressure, after the high pressure treatment. It can be seen that the position of the peak corresponding to $\tau_{4}$ in the classic processing is not shifted (near $4 \mathrm{~ns}$ ), but a tail extending up to $8 \mathrm{~ns}$ is added.

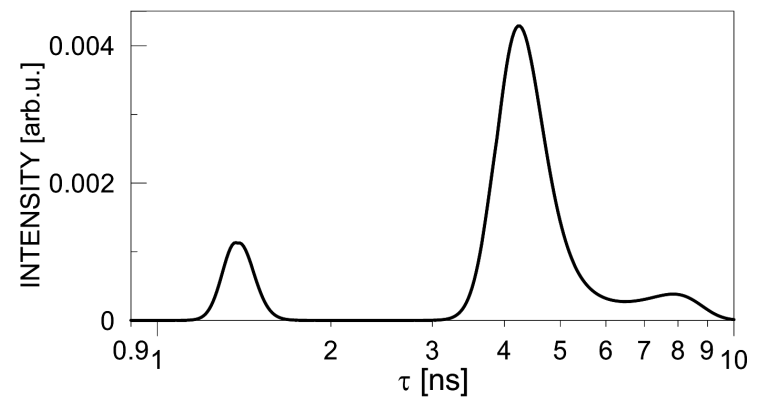

Fig. 3. The lifetime distributions of $o$-Ps components in PALS spectrum of CYTOP obtained by the MELT procedure at normal pressure after the cycle of measurements.

The sample, which before the measurements was transparent, after removal from the pressure chamber was found white, and fluffy. This can be the result of foaming at too fast reduction of pressure (in our compressor there is no possibility to reduce the pressure at a very low rate). However, after annealing at the temperature $470 \mathrm{~K}$ or more (i.e. well above the glass transition point), the sample structure was restored, the sample became transparent again and the PALS parameters returned to the values recorded before the pressure treatment.

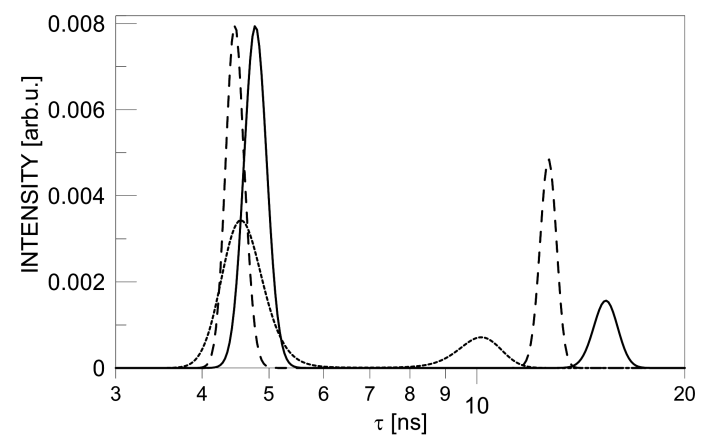

Fig. 4. The lifetime distributions of the two longest-living o-Ps components in PALS spectrum of CYTOP obtained by the MELT procedure at the pressure of nitrogen $30 \mathrm{MPa}$ (solid), $40 \mathrm{MPa}$ (dashed) and $70 \mathrm{MPa}$ (dotted line).

Several PALS spectra were measured with CYTOP in nitrogen atmosphere. The results were very similar to those in argon. The highest intensity of the longest component was about $4 \%$ at $40 \mathrm{MPa}$, that component disappeared above $80 \mathrm{MPa}$. For the three selected pressures, the PALS spectra were measured with high statistics and analyzed by both, the MELT and the LT programs. The MELT distributions for the two longest lifetime components (pores and "natural" free volumes) are shown in Fig. 4; respective lifetimes from the LT analysis are added in Figs. 1, 2.

\section{Conclusions}

The pressure dependence of the longest component (of pore origin) in CYTOP seems to be independent on the nature of gas used in the experiment; the pores are the effect of mechanical action of intruding gas. The best conditions of Ps formation in the gas filling the pores appear in a relatively narrow range of pressures around $\approx 35 \mathrm{MPa}$, at very high pressures the longest lifetime component disappears. After reduction of pressure, the lifetime $\tau_{4}$, describing the sizes of free volumes in the polymer, remains increased comparing to the sample before treatment. In addition to voids having the sizes as observed prior to the sample treatment also small number of larger voids appears.

\section{Acknowledgments}

Authors wish to thank T. Yoshimizu from Asahi Glass Co. and J. Pionteck from the Leibniz Institut für Polymerforschung in Dresden for supplying the samples of CYTOP

\section{References}

[1] X. Hong, Y.C. Jean, H. Yang, S.S. Jordan, W.J. Koros, Macromolecules 29, 7864 (1996).

[2] J. Bohlen, J. Wolff, R. Kirchheim, Macromolecules 32, 3766 (1999).

[3] J. Bohlen, R. Kirchheim, Macromolecules 34, 4210 (2001).

[4] A.Yu. Alentiev, V.P. Shantarovich, T.C. Merkel, V.I. Bondar, B.D. Freeman, Yu.P. Yampolskii, Macromolecules 35, 9513 (2002).

[5] W. Ma, A. Andersson, J. He, F.H.J. Maurer, Macromolecules 41, 5307 (2008).

[6] S. Claes, P. Vandezande, S. Mullens, M.K. VanBael, F.H.J. Maurer, Macromolecules 44, 2766 (2011).

[7] G. Dlubek, J. Pionteck, Yu Yang, S. Thränert, M. Elsayed, E. Badawi, R. Krause-Rehberg, Macromol. Chem. Phys. 209, 1920 (2008).

[8] G. Dlubek, J. Pionteck, M. Śniegocka, E.M. Hassan, R. Krause-Rehberg, J. Polym. Sci. Part B Polym. Phys. 45, 2519 (2007).

[9] J. Kansy, Nucl. Instrum. Methods Phys. Res. A 374, 239 (1996).

[10] A. Shukla, M. Peter, I. Hofmann, Nucl. Instrum. Methods Phys. Res. A 335, 310 (1993).

[11] M. Eldrup, D. Lightbody, J.N. Sherwood, Chem. Phys. 63, 51 (1981). 